\title{
The Magnaporthe oryzae Avirulence Gene AvrPiz-t Encodes a Predicted Secreted Protein That Triggers the Immunity in Rice Mediated by the Blast Resistance Gene Piz-t
}

\author{
Wei Li, ${ }^{1,3}$ Baohua Wang, ${ }^{2}$ Jun Wu, ${ }^{1}$ Guodong Lu, ${ }^{2}$ Yajun Hu, ${ }^{1}$ Xing Zhang, ${ }^{4}$ Zhengguang Zhang, ${ }^{4}$ \\ Qiang Zhao, ${ }^{1}$ Qi Feng, ${ }^{1}$ Hongyan Zhang, ${ }^{1}$ Zhengyi Wang, ${ }^{5}$ GuoLiang Wang, ${ }^{6}$ Bin Han, ${ }^{1}$ Zonghua Wang, ${ }^{2}$ \\ and Bo Zhou ${ }^{1,5}$ \\ ${ }^{1}$ National Center for Gene Research \& Institute of Plant Physiology and Ecology, Shanghai Institutes for Biological Sciences, \\ Chinese Academy of Sciences, Shanghai 200233 , China; ${ }^{2}$ Key Laboratory of Biopesticide and Chemical Biology, Ministry \\ of Education, Fujian Agriculture and Forestry University, Fuzhou 350002, China; ${ }^{3}$ Graduate School of the Chinese Academy \\ of Sciences, Beijing 100049, China; ${ }^{4}$ Department of Plant Pathology, Nanjing Agriculture University, Nanjing 210095, China; \\ ${ }^{5}$ Biotechnology Institute, Zhejiang University, Hangzhou 310029, China; ${ }^{6}$ Department of Plant Pathology, the Ohio State \\ University, Columbus 43210, U.S.A.
}

Submitted 24 September 2008. Accepted 17 November 2008.

The Magnaporthe oryzae avirulence gene AvrPiz-t activates immunity in a gene-for-gene fashion to rice mediated by the blast resistance gene Piz-t. To dissect the molecular mechanism underlying their recognition, we initiated the cloning of AvrPiz-t using a map-based cloning strategy. The AvrPiz-t gene was delimited to an approximately $21-\mathrm{kb}$ genomic fragment, in which six genes were predicted. Complementation tests of each of these six candidate genes led to the final identification of AvrPiz-t, which encodes a 108-amino-acid predicted secreted protein with unknown function and no homologues in M. oryzae or in other sequenced fungi. We found that AvrPiz-t is present in the virulent isolate GUY11 but contains a Pot 3 insertion at a position 462 bp upstream from the start codon. Complementation tests of AvrPiz-t genes driven by promoters of varying length revealed that a promoter larger than 462 bp is essential to maintain the AvrPiz-t function. These results suggest that a Pot3 insertion in GUY11 might interfere with the proper function of AvrPiz-t. Additionally, we found that AvrPiz-t can suppress the programmed cell death triggered by mouse BAX protein in Nicotiana benthamiana, identifying a mechanism by which AvrPiz-t may contribute virulence of M. oryzae.

In the long warfare between plants and phytopathogens, the plants have culminated in a highly effective immune system able to protect them from attack by a wide variety of pathogens. They have evolved two layers of immune responses referred to as pathogen-associated molecular pattern-triggered immunity (PTI) and effector-triggered immunity (ETI) (Chisholm et al.

W. Li and B. Wang contributed equally to this work.

Corresponding authors: Bo Zhou; Telephone: +86-21-54971317; Fax: +8621-64825775; E-mail: bzhou@ncgr.ac.cn; and Zonghua Wang; Telephone: +86-591-83790312; Fax: +86-591-83727618; E-mail: wangzh@ fjau.edu.cn

The genomic sequence of the AvrPiz-t gene was deposited at GenBank database (accession no. EU837058).

* The $\boldsymbol{e}$-Xtra logo stands for "electronic extra" and indicates that three supplementary tables are published online.
2006; Jones and Dangl 2006). The recognition of common features of microbial pathogens by diverse plant cell-surface receptors activates the PTI response to mount the primary defense. For example, both FLS2 and EFR can activate resistance to bacterial disease through perception of flagellin and EF-Tu, respectively (Zipfel et al. 2004, 2006). However, PTI can be effectively suppressed by so-called effector proteins encoded and delivered by phytopathogens into the plant cytosol. For example, several type III secretion system (T3SS) effectors from Pseudomonas syringae (e.g., HopM1, AvrPto, AvrRpm1, AvrRpt2, and HopAO1) were found to interfere with PTI in Arabidopsis (de Torres et al. 2006; He et al. 2006; Kim et al. 2005; Li et al. 2005; Nomura et al. 2006; Oh and Collmer 2005; Underwood et al. 2007). In order to monitor the presence of pathogen effectors, plants have developed the ETI, a more specialized immune response mediated by surveillance proteins, the so-called resistance $(\mathrm{R})$ proteins. The resistance activated by this pairwise association of $R$ and avirulence (Avr) genes was proposed as gene-for-gene resistance decades ago (Flor 1971). Only the coexistence of an $R$ gene and its cognate $A v r$ gene during interaction can activate the resistance, which is manifested as localized cell death (i.e., hypersensitive response [HR]). This response occurs at the site of infection to inhibit pathogen growth. The majority of $R$ genes in plants encode nucleotide-binding site leucine-rich repeat (NBS-LRR) proteins (Dangl and Jones 2001). Comparative genomics has demonstrated that all plants have large collections of NBS-LRR genes (McHale et al. 2006). Arabidopsis, for instance, maintains approximately 150 NBSLRR genes (Meyers et al. 2003).

It has been found that bacterial pathogens utilize a specialized T3SS to deliver the Avr proteins into the plant cytoplasm (Alfano and Collmer 2004; Grant et al. 2006). Most Avr genes in eukaryotic pathogens encode secreted proteins, including flax rust AvrL567, AvrM, AvrP4, and AvrP123 (Catanzariti et al. 2006; Dodds et al. 2004), AvrPi-ta in Magnaporthe oryzae (Orbach et al. 2000), Avrlb-1 in Phytophthora sojae (Shan et al. 2004), Avr3a in P. infestans (Armstrong et al. 2005), and ATR13 (Allen et al. 2004) and ATRl (Rehmany et al. 2005) in Hyaloperonospora parasitica. Furthermore, expression of these Avr genes inside the host cytoplasm was found to be 
essential for the activation of resistance mediated by their respective $R$ genes encoding NBS-LRR protein (Allen et al. 2004; Armstrong et al. 2005; Catanzariti et al. 2006; Dodds et al. 2004; Jia et al. 2000; Rehmany et al. 2005).

$M$. oryzae, the causal agent of the destructive blast disease to rice, is an ideal model with which to investigate the molecular mechanisms underlying the interaction between fungi and plants (Martin et al. 2002; Valent 1990). The recently completed genome sequences of both rice and $M$. oryzae have advanced the dissection of the molecular basis of genetic identification of both $R$ genes and $A v r$ genes (Dean et al. 2005; International Rice Genome Sequencing Project 2005). Currently, over 50 rice blast $R$ genes have been genetically analyzed, with 8 of them being characterized at the molecular level (Bryan et al. 2000; Chen et al. 2006; Lin et al. 2007; Liu et al. 2007; Qu et al. 2006; Wang et al. 1999; Zhou et al. 2006). Interestingly, only $\mathrm{Pi}$ - $\mathrm{d} 2$ encodes a non-NBS-LRR protein, suggesting that the majority of the blast resistance genes may encode NBSLRR R proteins (Chen et al. 2006; Dai et al. 2007). In the pathogen, more than $40 \mathrm{M}$. oryzae Avr genes have been genetically analyzed (Ma et al. 2006), with 5 being characterized at the molecular level. PWL1 and PWL2 both confer resistance specificity to weeping lovegrass and encode sequence-related proteins without ascribed function (Kang et al. 1995; Sweigard et al. 1995). Avrl-CO39 was confined to a $1.05-\mathrm{kb}$ region containing several potential open reading frames (ORF) (Farman et al. 2002). Avr-Pita encodes a 223-amino-acid (aa) polypeptide with features typical of metalloproteases and was found to trigger $P i$ - $t a$-specific immunity inside rice cells (Orbach et al. 2000). ACE1 encodes a large cytoplasmic enzyme of 4,035 aa that is unlikely to be secreted and was found to be required for triggering the Pi33-specific resistance response (Böhnert et al. 2004). Of these blast Avr genes, PWL1, PWL2, and Avr-Pita encode secreted proteins, suggesting that the secretion is required for their Avr function (Jia et al. 2000; Kang et al. 1995; Orbach et al. 2000).

Despite significant progress on the genetic analyses of both $R$ genes and $A v r$ genes in the rice blast pathosystem, only one matched pair of $R$ and Avr genes, Pi-ta/Avr-Pita, has been analyzed in the rice blast pathosystem (Bryan et al. 2000; Jia et al. 2000; Orbach et al. 2000). The Pi-ta gene encodes a predicted cytoplasmic receptor with a centrally localized NBS and C-terminal leucine-rich domain (LRD), while Avr-Pita encodes a putative metalloprotease (Bryan et al. 2000; Orbach et al. 2000). Both Pita and Avr-Pita were found to interact physically by both yeast-two hybrid experiments and an in vitro assay (Jia et al. 2000). To elucidate more deeply the molecular mechanism underlying the interaction between $R$ genes and $A v r$ genes, more $R / A v r$ pairs will need to be studied.

Both $R$ genes Pi2 and Piz-t encode highly related NBS-LRR proteins and confer resistance to an overlapping but different set of diverse $M$. oryzae strains (Zhou et al. 2006). Only 8 aa differences exclusively confined within three consecutive LRR distinguish Pi2 from Piz-t, indicating that the LRR domain is a primary specificity determinant (Zhou et al. 2006). M. oryzae Avr gene AvrPiz- $t$ recognized by Piz- $t$ was previously mapped between two markers, MAT1 and $\mathrm{CH} 5-75 \mathrm{H}$, on chromosome 7 (Luo et al. 2005). The final cloning of AvrPiz- $t$ will be helpful in unraveling the molecular mechanism underlying the recognition between Piz-t and AvrPiz-t . In this study, we describe the isolation of AvrPiz-t using a map-based cloning strategy.

\section{RESULTS}

\section{Map-based cloning of AvrPiz-t.}

An $\mathrm{F}_{1}$ population consisting of 137 single ascospores derived from a cross between avirulent isolate 81278ZB15
(MAT1-1) collected from a field in Fujian province, China and virulent isolate GUY11 (MAT1-2) was constructed for the genetic analysis of AvrPiz-t. Of the 52 single ascospores tested, 29 progeny were avirulent to transgenic rice plants carrying Piz- $t$ whereas 23 were virulent. The segregation ratio of a virulence versus virulence was found to fit a $1: 1$ ratio in the $\chi^{2}$ test of a goodness-of-fit $\left(\chi^{2}=0.69, P=0.4\right)$, indicating that AvrPiz- $t$ is controlled by a single locus.

A polymerase chain reaction (PCR)-based marker system to distinguish between the presence or absence of a transposable element (PATE) was developed in this study (discussed below). Using this system, we identified a total of 11 PATE markers showing polymorphisms on chromosome 7 between two parental isolates (Table 1). AvrPiz-t was first mapped between two PATE markers, P21.1698.1 and P4.520.1, and was found to co-segregate with another two PATE markers, P21.1709.1 and P21.1709.2 (Fig. 1A; Table 1). Another two single nucleotide polymorphism (SNP) markers, SNP1702 and SNP514, derived from transposable elements $\mathrm{P} 21.1702 .1$ and $\mathrm{P} 4.514 .1$, respectively, were used to further narrow the location of AvrPiz-t (Fig. 1A; Supplementary Table 1). By aligning the sequences of SNP1702 and SNP514 on the sequence of chromosome 7 (GenBank: CM000230), we found that the genomic region between these two markers is approximately $145 \mathrm{~kb}$. Therefore, the construction of a BAC contig that covers the AvrPiz- $t$ locus in avirulent parental isolate $81278 \mathrm{ZB} 15$ was initiated (Fig. 1B). One BAC clone, BAC07bg07, was then completely sequenced. Fine mapping of AvrPiz-t finally confined it to an approximately $21-\mathrm{kb}$ genomic interval flanked by two SNP markers, SNP12 and SNP78 (Fig. 1C). In all, six genes, designated $7 \mathrm{bg} 7.12$ to $7 \mathrm{bg} 7.17$, were predicted in this region (Fig. 1D). The colinear regions containing these genes were amplified from the virulent parental isolate GUY11 and their sequence was determined. It was found that the coding and promoter sequence of both $7 \mathrm{bg} 7.12$ and $7 \mathrm{bg} 7.17$ in GUY11 were identical to the ones in $81278 \mathrm{ZB} 15$, suggesting that neither of them could be the Piz-t candidate. In the remaining four genes, sequence polymorphisms were identified in either coding or promoter sequence between 81278ZB15 and GUY11 (Supplementary Table 2).

\section{Determination of $A v r P i z-t$ by gene complementation tests.}

In order to identify the AvrPiz-t candidate from these four genes, gene complementation tests were conducted, where each gene was transformed into GUY11. Pathogenicity reactions of the derived transformants were analyzed using both susceptible rice cv. Nipponbare and Piz-t-transgenic Nipponbare. It was found that only transformants into which $7 \mathrm{bg} 7.15$ was introduced showed avirulence to Piz-t-transgenic Nipponbare (Fig. 2; Table 2). Nevertheless, the transformants derived from the other three genes showed virulence, suggesting that they did not receive AvrPiz-t (Fig. 2; Table 2). Based on the pathogenicity reaction test, all the transformants showed virulence to Nipponbare. Therefore, it has been clearly demonstrated that the Piz-t-mediated resistance requires the introduction of $7 \mathrm{bg} 7.15$.

\section{AvrPiz-t encodes a predicted secreted protein.}

AvrPiz- $t$ is an intronless gene of 327 bp in length and encodes a predicted 108-aa polypeptide. It is a single copy gene in the 70-15 genome confirmed by homology search. The deduced AvrPiz-t product shows no substantial homology to any known proteins in $M$. oryzae as well as in any other organism. An 18-aa secretion signal was identified at the aminoterminus by using both SignalP 3.0 and ProtComp programs. In order to further demonstrate the importance of this N-terminal secretion signal, a construct, designated pAvrPiz- $t_{90}$, 
was created by removal of the secretion signal (Table 2). The derived construct was then transformed into virulent isolate GUY11. Pathogenicity tests of the transformants showed that they were virulent to Piz-t-transgenic rice, indicating that AvrPiz- $t_{90}$ is no longer capable of triggering immunity medi- ated by Piz-t (Table 2). Therefore, it was concluded that the N-terminal secretion signal is critical for the Avr function of AvrPiz-t. However, it is unknown whether removal of the secretion signal makes AvrPiz- $\mathrm{t}_{90}$ unable to be mobilized outside the cell or not.

Table 1. Distribution of transposable elements (TE) and presence or absence of a TE (PATE) markers on chromosome 7 of Magnaporthe oryzae $e^{\mathrm{a}}$

\begin{tabular}{|c|c|c|c|c|c|c|}
\hline Supercontig, contig & Contig length (bp) & TE location (bp) & TE length (bp) & Primer sequences $^{\mathrm{b}}$ & PATE designation & Polymorphism \\
\hline \multicolumn{7}{|l|}{25} \\
\hline 1829 & 21,909 & $2,081-3,944$ & 1,864 & $\ldots$ & $\ldots$ & $\ldots$ \\
\hline 1842 & 14,880 & $8,898-10,760$ & 1,863 & & & $\ldots$ \\
\hline \multirow{4}{*}{1850} & 51,013 & $2,715-4,481$ & 1,767 & F gcagtgacaacctttgc & P25.1850.1 & Yes \\
\hline & $\ldots$ & & & $\mathrm{R}$ cgtcgtcgtaatcacac & & \\
\hline & $\ldots$ & $15,111-16,978$ & 1,868 & F ttgcatcctaccagctgc & P25.1850.2 & Yes \\
\hline & & & & $\mathrm{R}$ ttcaacccccaggcagac & & \\
\hline \multirow[t]{2}{*}{1856} & 27,674 & $16,319-18,185$ & 1,867 & F aaaggcagaggcattgtg & $\mathrm{P} 25.1856 .1$ & Yes \\
\hline & $\ldots$ & $\ldots$ & $\ldots$ & R cggtgcagacatttactg & $\ldots$ & $\ldots$ \\
\hline \multicolumn{7}{|c|}{1.00 .0 . } \\
\hline \multirow[t]{2}{*}{2031} & 37,033 & $2,472-2,949$ & 478 & $\mathrm{~F}$ attgtacacatcactcc & P37.2031.1 & Yes \\
\hline & $\ldots$ & $\ldots$ & $\ldots$ & $\mathrm{R}$ caagctcaactcgaagc & $\ldots$ & $\ldots$ \\
\hline \multicolumn{7}{|l|}{21} \\
\hline \multirow[t]{2}{*}{1698} & 30,783 & $23,589-25,451$ & 1,863 & F ttgcttgcagccattgac & P21.1698.1 & Yes \\
\hline & & & & $\mathrm{R}$ cataacaatgtcccactc & & \\
\hline \multirow[t]{2}{*}{1702} & 9,614 & $3,914-5,773$ & 1,860 & $\mathrm{~F}$ atccgacctaatcgtgac & $\mathrm{P} 21.1702 .1$ & No \\
\hline & $\ldots$ & & & $\mathrm{R}$ acggctgcaatcatgaag & $\ldots$ & $\ldots$ \\
\hline 1703 & 5,193 & $3,040-4,904$ & 1,865 & $\ldots$ & $\ldots$ & $\ldots$ \\
\hline 1705 & 5,505 & $2,394-4,257$ & 1,864 & $\ldots$ & $\ldots$ & $\ldots$ \\
\hline \multirow[t]{4}{*}{1709} & 21,702 & $6,512-8,382$ & 1,871 & F gaaggtcattttctcacag & P21.1709.1 & Yes \\
\hline & $\ldots$ & & & $\mathrm{R}$ atccaattcatgaacacgac & 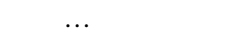 & $\ldots$ \\
\hline & $\ldots$ & $11,736-13,601$ & 1,866 & F taacctgtcaacgaagatag & P21.1709.2 & Yes \\
\hline & & & & $\mathrm{R}$ aagtgacgggatttggttc & $\ldots$ & $\ldots$ \\
\hline 1713 & 2,908 & $715-1,169$ & 455 & $\ldots$ & $\ldots$ & $\ldots$ \\
\hline 1719 & 16,164 & $2,472-5,167$ & 2,696 & $\ldots$ & $\ldots$ & $\ldots$ \\
\hline 1720 & 19,931 & $8,502-8,963$ & 462 & $\ldots$ & $\ldots$ & $\ldots$ \\
\hline 1723 & 25,973 & $17,430-19,295$ & 1,866 & $\ldots$ & $\ldots$ & $\ldots$ \\
\hline 1725 & 20,877 & $6,663-8,497$ & 1,835 & $\ldots$ & $\ldots$ & $\ldots$ \\
\hline 1738 & 10,202 & $5,518-5,967$ & 450 & $\ldots$ & $\ldots$ & $\ldots$ \\
\hline \multirow[t]{2}{*}{1739} & 23,489 & $7,832-9,698$ & 1,867 & F cggagagggaatggatc & P21.1739.1 & No \\
\hline & & & & $R$ gaaaagctgcgtaaagg & $\ldots$ & $\ldots$ \\
\hline \multicolumn{7}{|l|}{4} \\
\hline \multirow[t]{2}{*}{514} & 8,058 & $3,670-5,532$ & 1,863 & F ttgcactgggtaccgttacc & P4.514.1 & No \\
\hline & $\cdots$ & & & $\mathrm{R}$ atgcgtgaaaccaaactcgg & & \\
\hline \multirow[t]{2}{*}{520} & 36,000 & $14,769-16,633$ & 1,865 & F ttcgatggcagtgctggag & P4.520.1 & Yes \\
\hline & & & & $\mathrm{R}$ tatgcaaggaggatggcttg & $\ldots$ & $\ldots$ \\
\hline 522 & 32,999 & $603-2,467$ & 1,865 & $\ldots$ & ( & $\ldots$ \\
\hline 523 & 72,540 & $35,372-37,258$ & 1,887 & F actctatggaatcgctgctc & P4.523.1 & Yes \\
\hline & $\ldots$ & & & $\mathrm{R}$ aagttcaggtcatctggtag & $\ldots$ & $\ldots$ \\
\hline & $\ldots$ & $42,640-44,504$ & 1,865 & F ctcctctagctatctacatg & P4.523.2 & Yes \\
\hline & $\ldots$ & & (1) & $\mathrm{R}$ aaggtcagactaatagtgcc & $\ldots$ & $\ldots$ \\
\hline 527 & 27,274 & $7,598-7,726$ & 129 & $\ldots$ & $\ldots$ & $\ldots$ \\
\hline 537 & 21,003 & $18,106-18,227$ & 122 & & & \\
\hline 542 & 21,366 & $19,597-19,720$ & 124 & F caagcggtgtcttttatgag & P4.542.1 & No \\
\hline & & & & $R$ atctgacgtgaccattgtag & & \\
\hline 557 & 35,303 & $19,492-19,647$ & 156 & F gaggatgtcatcgaagag & P4.557.1 & No \\
\hline & & & & $\mathrm{R}$ catgtctcgacacattcc & & \\
\hline 561 & 92,635 & $2,042-3,904$ & 1,863 & $\mathrm{~F}$ ataccggtatgccaatcg & P4.561.1 & Yes \\
\hline & $\ldots$ & & & $\mathrm{R}$ actccaatccgttaagcc & $\ldots$ & $\ldots$ \\
\hline & $\ldots$ & $63,542-64,000$ & 459 & F cagcttgtataagagagg & P4.561.2 & No \\
\hline & 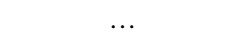 & & & $\mathrm{R}$ aggtatttgttggatcgc & $\ldots$ & $\ldots$ \\
\hline 565 & 32,557 & $5,460-5,587$ & 128 & $\ldots$ & $\ldots$ & $\ldots$ \\
\hline 581 & 10,725 & $7,535-7,994$ & 460 & $\ldots$ & $\ldots$ & $\ldots$ \\
\hline 595 & 60,696 & $53,111-55,802$ & 2,692 & $\ldots$ & $\ldots$ & $\ldots$ \\
\hline 600 & 80,506 & $9,714-12,404$ & 2,691 & $\ldots$ & $\ldots$ & $\ldots$ \\
\hline 605 & 56,017 & $39,488-39,617$ & 130 & $\ldots$ & $\ldots$ & $\ldots$ \\
\hline 608 & 8,622 & $7,295-8,148$ & 854 & $\ldots$ & $\ldots$ & $\ldots$ \\
\hline 611 & 13,828 & $4,751-7,442$ & 2,692 & $\ldots$ & $\ldots$ & $\ldots$ \\
\hline 32 & & & & & & \\
\hline 1963 & 16,649 & $8,252-10,115$ & 1,864 & $\ldots$ & $\ldots$ & $\ldots$ \\
\hline 33 & & & & & & \\
\hline 1983 & 57,283 & $10,163-10,621$ & 459 & $\ldots$ & $\ldots$ & $\ldots$ \\
\hline & & $52,183-52,630$ & 448 & $\ldots$ & $\ldots$ & $\ldots$ \\
\hline 1990 & 33,944 & $12,320-14,136$ & 1,817 & $\ldots$ & $\ldots$ & $\ldots$ \\
\hline
\end{tabular}

${ }^{a}$ Release 2, not 5, of the M. oryzae genome assembly (Magnaporthe grisea database) was used for sequence analysis because there is some misassembly found in release 5 .

${ }^{\mathrm{b}} \mathrm{F}=$ forward and $\mathrm{R}=$ reverse. 
The Pot3 insertion disturbs the proper function of the promoter of AvrPiz-t.

Sequence comparison of the AvrPiz-t gene between isolates $81278 Z$ Z15 and GUY11 revealed no sequence variation in their coding regions. However, a Pot 3 transposon is located at a position $462 \mathrm{bp}$ upstream from the start codon of AvrPiz- $t$ in GUY11 (Fig. 3A). It is 1,861 bp in length and contains two 48bp inverted terminal repeats (ITR) and one ORF exhibiting significant homology to transposases encoded by TC1/Mariner transposons (Farman et al. 1996). Moreover, it shares 99\% identity in nucleotide sequence to the one reported in the AvrPita locus (GenBank: AF333034) (Kang et al. 2001). Transposition of Pot3 in AvrPiz-t in GUY11 resulted in the duplication of the target sequence ( $5^{\prime}$ TA $\left.3^{\prime}\right)$. After removal of Pot3 and one copy of the target sequence, the promoter sequence of AvrPiz-t in GUY11 was found to be identical to the one in 81278ZB15.

To determine the minimum size of promoter which is required for the proper function of AvrPiz-t, we made four constructs, including one mimicking the version in GUY11, and transformed them into GUY11. It was found that both pP537 and pP736 constructs, but neither pP454 nor pP462, were able to complement the AvrPiz-t function (Fig. 3B; Table 2). These results indicated that the minimum size of the promoter to properly maintain the function of AvrPiz- $t$ should be larger than $462 \mathrm{bp}$, the position at which Pot3 inserted in GUY11. Therefore, sequences essential for AvrPiz- $t$ function are located upstream from the insertion site. The transcription of AvrPiz- $t$ in both $81278 \mathrm{ZB} 15$ and GUY11 at mycelium, conidium, appressorium, and in planta stages was investigated to further validate the hypothesis. However, due to undetectable levels of expression of AvrPiz-t by reverse-transcription PCR in the conditions tested, we were not able to demonstrate whether the insertion of Pot3 interferes with the expression of AvrPiz-t in GUY11.

\section{Virulent isolates exhibit either a Pot3 insertion or amino acid substitution in AvrPiz-t.}

To investigate the sequence variation and resulting phenotypes of AvrPiz-t in diverse $M$. oryzae isolates, we analyzed the AvrPiz- $t$ sequence in nine virulent and avirulent isolates (Table 3). It was found that no sequence changes existed in AvrPiz- $t$ in the avirulent isolates GDS2 and KJ201 (Table 3). However, two types of sequence variations were revealed in the virulent isolates. AvrPiz- $t$ in the isolate 70-15 had the same Pot3 insertion in its promoter region as the one identified in GUY11 (Table 3). In the remaining six isolates, as shown in Table 3, AvrPiz-t had the same single nucleotide substitution ( $\mathrm{C}$ to $\mathrm{T}$ ), resulting in an amino acid change of valine for alanine at position $41(\mathrm{~A} 41 \mathrm{~V})$. It is worthwhile to note that all the six virulent isolates might have originated from the same isolate because they all came from the same province in China. In order to further confirm that substitution A41V caused the loss of function of AvrPiz-t, the pAvrPiz-t ${ }^{41 \mathrm{~V}}$ allele from the

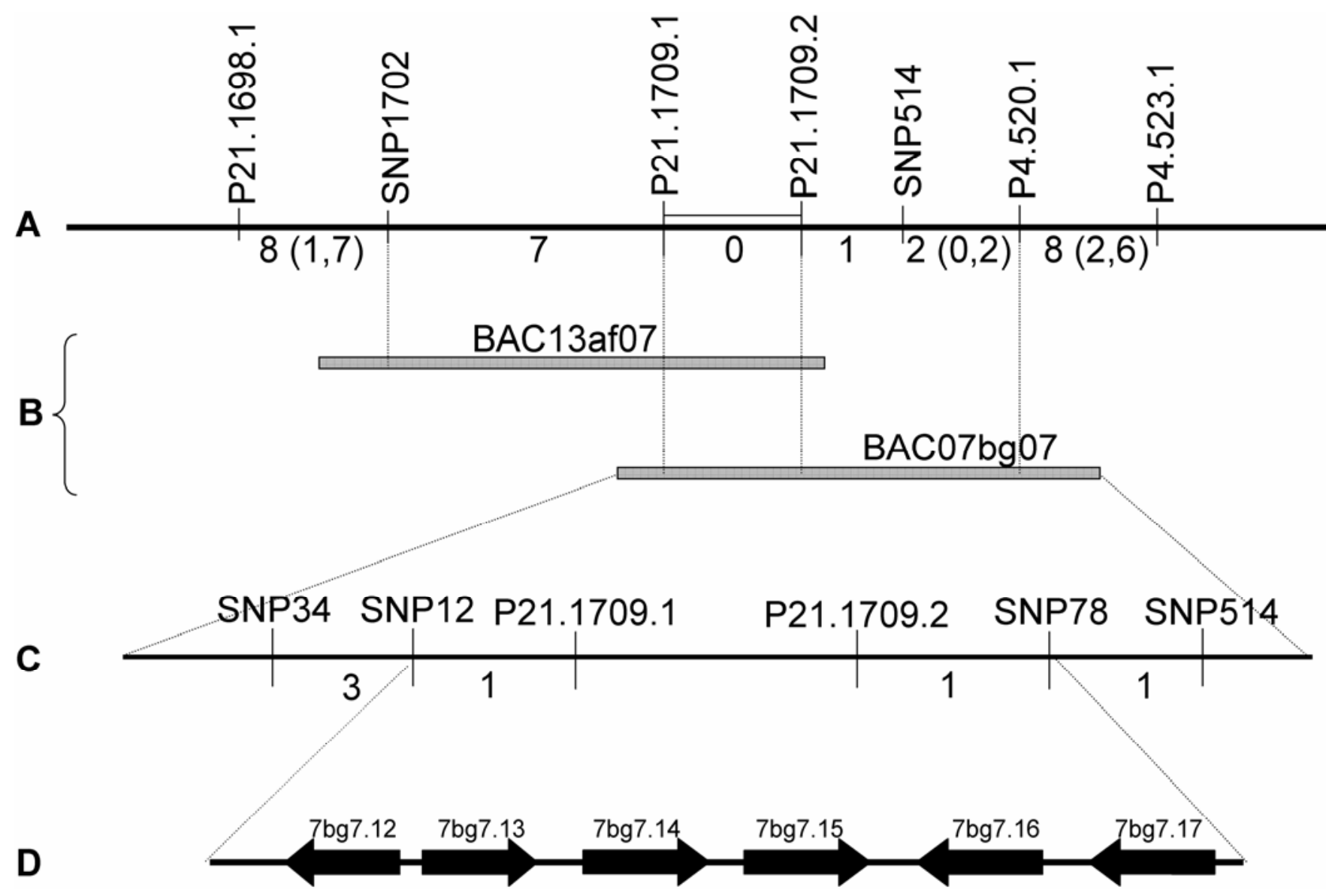

Fig. 1. Map-based cloning of AvrPiz-t. A, Genetic analysis of AvrPiz-t. Molecular markers are listed above the string and the corresponding recombinants from AvrPiz- $t$ are listed underneath. The number of the recombinants at P21.1698.1, P4.520.1, and P4.523.1 derived from two rounds of mapping analyses as described in Materials and Methods is indicated in parenthesis. B, Physical mapping of AvrPiz-t. The BAC contig spanning the AvrPiz-t locus consisted of two BAC clones indicated in gray rectangles with their names above. C, Fine mapping of AvrPiz- $t$. Molecular markers are listed above the string and corresponding recombinants from AvrPiz- $t$ are listed underneath. D, Candidate genes for AvrPiz- $t$. Genes are represented by filled arrows indicating direction of transcription. The gene designation is listed above the arrows. The figure is not drawn to scale. 
virulent strain, GDS3, was transformed into GUY11 (Table 2). Transformants were found to be virulent to Piz-t, further indicating that AvrPiz-t ${ }^{41 \mathrm{~V}}$ was not able to produce the AvrPiz-t phenotype (Tables 2 and 3). Taken together, both insertion of Pot3 and the substitution A41V can cause the loss of function of AvrPiz-t.

\section{AvrPiz-t can suppress the cell death induced by BAX in tobacco.}

Many bacterial and filamentous pathogen effectors were found to be able to suppress programmed cell death (PCD) induced by the proapoptotic protein BAX, identifying a mechanism by which these effectors contribute positively to the virulence of pathogens (Dou et al. 2008; Jamir et al. 2004). We tested whether AvrPiz-t could also suppress BAX-triggered PCD in Nicotiana benthamiana. Obvious cell death symptoms were observed when $N$. benthamiana leaves were infiltrated with Agrobacterium tumefaciens cells carrying pGR106:BAX (Fig. 4A). Filtration of $N$. benthamiana leaves with $A$. tumefaciens cells containing a green fluorescent protein (GFP) gene (Fig. 4B) or $\mathrm{MgCl}_{2}$ buffer (data not shown) did not affect the BAX-triggered cell death. Infiltration of $N$. benthamiana leaves with A. tumefaciens cells harboring AvrPiz-t alone did not cause obvious cell death symptoms (Fig. 4C).
However, we found that $N$. benthamiana leaves infiltrated with A. tumefaciens cells harboring the AvrPiz-t gene $24 \mathrm{~h}$ prior to infiltration with the pGR106:BAX-harboring cells did not produce symptoms (Fig. 4D). The expression of BAX was detected at $24 \mathrm{~h}$ and later, after infiltration, which ruled out the possibility of expression failure of BAX in tests (data not shown). These results indicated that AvrPiz-t could suppress the BAX-mediated PCD in N. benthamiana.

\section{DISCUSSION}

In this study, we described our effort to clone the $M$. oryzae Avr gene AvrPiz-t, complementary to the previously cloned rice blast resistance gene Piz- $t$ (Zhou et al. 2006), using a mapbased cloning strategy. The Piz-t/AvrPiz-t pair is the second $R / A v r$ pair in the rice and rice blast system to be characterized at the molecular level. The cloning of AvrPiz-t as well as Piz-t will be very helpful in gaining insight into mechanisms of rice resistance to $M$. oryzae.

In the last decade, over $20 \mathrm{Avr}$ genes have been isolated from filamentous microorganisms, including fungi and oomycetes (Kamoun 2007). Most of them encode small secreted proteins without known functions (Kamoun 2006). In $M$. oryzae, four Avr genes-Avr-Pita (Orbach et al. 2000) ACE1
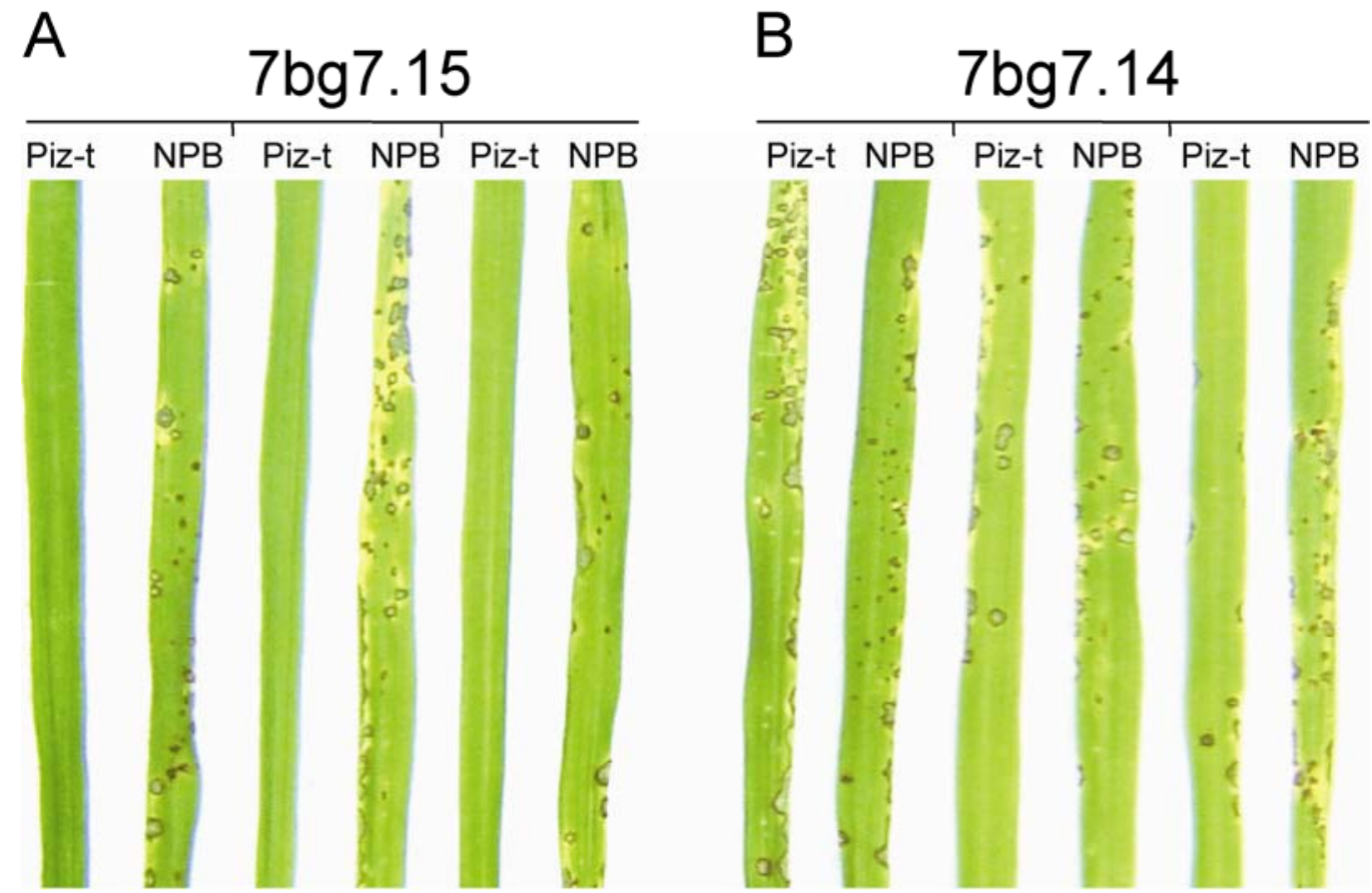

Fig. 2. Complementation tests of AvrPiz-t. Pathogenicity tests of strains transformed with two candidate genes, $7 \mathrm{bg} 7.15 \mathrm{or} 7 \mathrm{bg} 7.14$, were used for illustration. Results for three independent transformed isolates for each candidate gene are illustrated from left to right. The photos were taken 7 days after inoculation. NPB, Nipponbare; Piz-t, Nipponbare containing Piz-t transgene. A, GUY11 transformants with $7 \mathrm{bg} 7.15$ are virulent to Nipponbare but avirulent to Nipponbare containing Piz-t transgene. B, GUY11 transformants with $7 \mathrm{bg} 7.14$ are virulent to both Nipponbare and Nipponbare containing Piz-t $t$ transgene.

Table 2. All the key constructs used in the study

\begin{tabular}{llc}
\hline Constructs $^{\text {a }}$ & \multicolumn{1}{c}{ Description } & Phenotype \\
\hline p7bg7.13 & Entire region of the predicted gene 7bg7.13 & Virulent \\
p7bg7.14 & Entire region of the predicted gene 7bg7.14 & Virulent \\
p7bg7.15 & Entire region of the predicted gene 7bg7.15 & Avirulent \\
p7bg7.16 & Entire region of the predicted gene 7bg7.16 & Virulent \\
pAvrPiz-t ${ }_{90}$ & AvrPiz-t without N-terminal 18-amino-acid secreted signal & Virulent \\
pP454 & AvrPiz-t driven by a 454-bp promoter & Virulent \\
pP462 & AvrPiz-t in GUY11 containing a portion of Pot3 at the position of 462-bp promoter site & Virulent \\
pP537 & AvrPiz-t driven by a 537-bp promoter & Avirulent \\
pP736 & AvrPiz-t driven by a 736-bp promoter & Avirulent \\
pAvrPiz-t ${ }^{41 \mathrm{~V}}$ & AvrPiz-t carrying a single amino acid change (A to V) at position 41 & Virulent \\
\hline
\end{tabular}


(Böhnert et al. 2004), Avrl-CO39 (Farman et al. 2002; Peyyala and Farman 2006), and AvrPiz-t in this study - complementary to different rice blast resistance genes have been cloned. Both Avr-Pita and Avrl-CO39 encode predicted secreted proteins (Orbach et al. 2000; Peyyala and Farman 2006). AvrPiz- $t$ described in this study also encodes a small protein predicted to be secreted. Taken together, we speculated that the major repertoire of $M$. oryzae Avr genes might encode secreted proteins.

It has been found that more than $9.7 \%$ of the $M$. oryzae genome is made up of repetitive DNA based on the analysis of the whole-genome sequence (WGS) of 70-15 (Dean et al. 2005). Even a higher proportion (nearly 14\%) of the repetitive DNA composition was identified when using the combined BAC-WGS sequence of chromosome 7 of 70-15 (Thon et al. 2006). Moreover, virtually all of the repetitive DNA sequences found on chromosome 7 consisted of transposable elements (TE), which were distributed as clusters (Thon et al. 2006). The abundance of the TE has been implicated as a major contributor to genome rearrangements and as a source of genetic variations (Nishimura et al. 2000; Kang et al. 2001). Here, we took advantage of polymorphisms of TE with respect to their presence and absence in parental isolates and designed PATE markers for genetic analysis of AvrPiz-t. A significant portion (11 of 17) of the tested PATE showed polymorphisms between parental strains (Table 1), suggesting that polymorphisms with respect to presence or absence of TE are significant among diverse $M$. oryzae strains. In addition to other DNA-based markers associated with TE, (e.g., rep-PCR and retrotransposon-microsatellite amplified polymorphism) (Chadha and Gopalakrishna 2005; Wang et al. 2002), the PATE marker system described herein provided another tool for genetic analysis of M. oryzae.

It has been reported that $M$. oryzae has relatively more genetic instability in Avr determinants corresponding to certain rice blast $R$ genes, making the utility of $R$ genes in controlling rice blast disease limited (Bonman et al. 1987; Kiyosawa 1976). However, mechanisms underlying the instability of Avr determinants in $M$. oryzae are largely unknown. It is compelling that TE were always found to be associated with the loss of function or rearrangement of $M$. oryzae $A v r$ genes (i.e., Avrl-CO39, Avr-Pita, and ACE1) (Böhnert et al. 2004; Farman et al. 2002; Kang et al. 2001). An unknown retrotransposon and a repetitive element were identified at right- and left-hand borders, respectively, of deletion breakpoints of the AvrlCO39 locus in GUY11 as well as in other virulent isolates (Farman et al. 2002). An insertion of the $M G L$ line-like retroposon was identified in the last exon of $A C E 1$, which caused a loss of function in the virulent isolate 2/0/3 (Böhnert et al. 2004). A Pot 3 insertion was identified in the promoter of $A v r$ Pita, which caused the gain of virulence in the mutant strain CP1632 (Kang et al. 2001). Zhou and associates (2007) identified a Pot3 transposon in the DNA region encoding the putative protease motif of the Avr-Pita protein from field-virulent isolate B2. Analyses of the genomic organization and evolution of the Avr-Pita gene family further showed family members to be closely associated with diverse TE, including ITR transposons, retroposons, solo long-terminal repeats (LTR), and undefined short repetitive DNA elements (Khang et al. 2008). Here, we found that a Pot 3 insertion in the promoter region caused the loss of function of AvrPiz-t, providing another example for the association of TE with $A v r$ genes. It is reasonable to hypothesize that TE function as an on/off switch controlling the expression of Avr genes for defeating rice resistance genes in nature. Given that the $M$. oryzae genome encodes a large number of effectors contributing accumulatively to virulence, loss of function of a particular Avr gene does not compromise the entire virulence phenotype of $M$. oryzae. However, loss of function of a particular Avr gene by insertion of a TE will make a strain unrecognized by rice cultivars harboring the complementary $R$ gene. Therefore, insertion of TE in $A v r$ genes may possibly be one of the strategies that $M$. oryzae has evolved to maintain high instability in Avr determinants in the field.

Table 3. Sequence variation of the AvrPiz-t gene in different isolates

\begin{tabular}{lcl}
\hline Isolates & Sequence variation & Phenotype \\
\hline KJ201 & No & Avirulent \\
$70-15$ & Insertion of Pot3 & Virulent \\
GDS2 & No & Avirulent \\
GDS3 & A single SNP & Virulent \\
GDS4 & A single SNP & Virulent \\
GDS6 & A single SNP & Virulent \\
GDS8 & A single SNP & Virulent \\
GDS10 & A single SNP & Virulent \\
GDS11 & A single SNP & Virulent \\
\hline
\end{tabular}

${ }^{\text {a }} \mathrm{SNP}=$ single nucleotide polymorphism
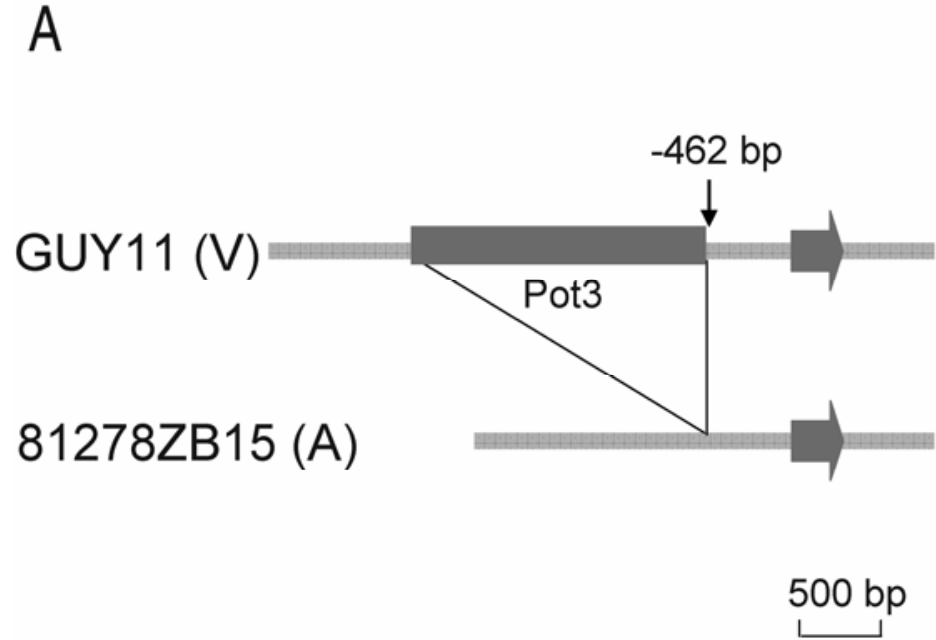
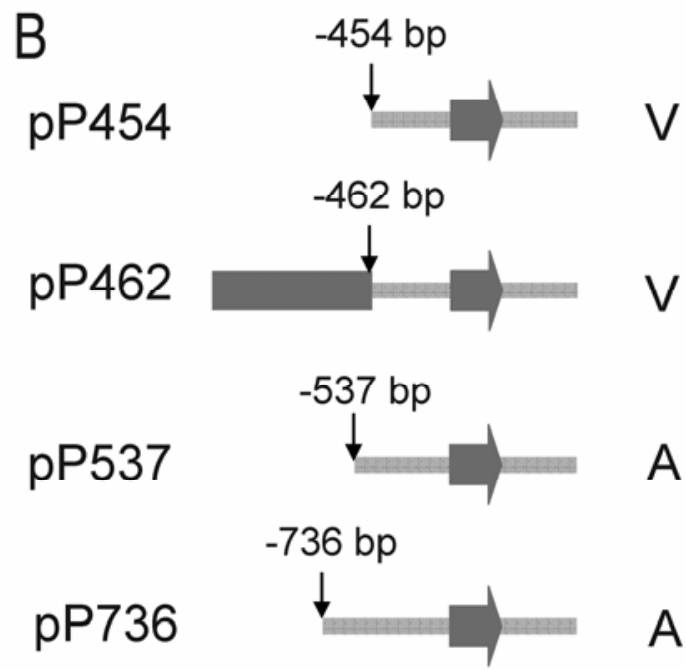

Fig. 3. Schematic determination of the promoter size required for proper function of AvrPiz-t. AvrPiz-t is indicated by filled arrows and Pot 3 is indicated by a filled rectangle. The position at which the Pot3 inserted is marked with vertical arrows and base pair positions. A, Pot3 located at 462 bp upstream from start codon (ATG) of AvrPiz- $t$ in the virulent isolate GUY11. B, Pathogenicity tests of each strain transformed with four AvrPiz- $t$ variants with different size of promoter. The name of each construct is listed at the left and the phenotype to Piz-t transgenic rice plants of the derived transformants is at the right. A: avirulent; V: virulent. 


\section{MATERIALS AND METHODS}

Fungal isolates and rice cultivars used in this study.

The M. oryzae isolate $81278 \mathrm{ZB} 15$ that is avirulent to Piz-tharboring rice cultivars was collected from a field in Fujian province, China. It was used as one of the parental isolates for $F_{1}$ population construction. The isolate GUY11 that is virulent to Piz-t-harboring rice cultivars was used as another parental isolate. It was also used as the recipient isolate for M. oryzae transformation experiments in this study. To create the population, 137 single ascospores were randomly picked and rescued for genetic analysis. Rice cv. Nipponbare and a Piz-t transgenic rice line in Nipponbare background were used for the pathogenicity tests. The Piz-t donor line IRBL11 and susceptible rice cv. CO39 were also used as control rice cultivars for the pathogenicity tests.

\section{Pathogenicity tests and disease assessment.}

Infection assays were conducted on 21-day-old rice seedlings and the resulting resistance or susceptible phenotype was evaluated 7 days after inoculation as described previously (Zhou et al. 2006). The pathogenicity tests for parental, progeny, and transformant isolates were repeated two times.

\section{The development of PATE markers for genetic analysis.}

A set of molecular markers representing PATE in two parental strains were developed for genetic analysis. TE with typical features (e.g., ITR, LTR, and target duplicated sequences) pre- sent in the sequence of chromosome 7 of M. oryzae were identified by BLAST search. In order to make the PATE suitable for molecular analysis, we selected TE which were smaller than $3 \mathrm{~kb}$ in size. Primers were then designed from the flanking sequences of TE. PCR-amplified products were analyzed for polymorphisms regarding the presence or absence of the TE between two parental isolates. Verified polymorphic PATE were used for the genetic analysis.

\section{Genetic analysis of AvrPiz-t.}

We adopted two rounds of genetic analyses to delimit the location of the AvrPiz-t gene. At the first round, we mapped AvrPiz- $t$ between two markers, P21.1698.1 and P4.523.1, at which one and two recombinants, respectively, were identified from 52 progeny isolates. At the second round, we identified 13 recombinants between these two markers at the DNA level from the remaining 85 progeny isolates. After phenotype evaluation, seven and six more recombinants at P21.1698.1 and P4.523.1, respectively, were counted. Therefore, we used these 16 recombinants to further narrow down the location of AvrPiz-t. Two SNP markers, SNP514 and SNP1702, developed based on the reference sequence of 70-15 helped to delimit AvrPiz- $t$ to an approximately $145-\mathrm{kb}$ genomic interval. Both BAC contig construction and sequence determination of the AvrPiz- $t$ locus facilitated the development of more SNP markers. AvrPiz- $t$ was finally delimited into an approximately $21-\mathrm{kb}$ genomic interval flanked by two SNP markers, SNP12 and SNP78.
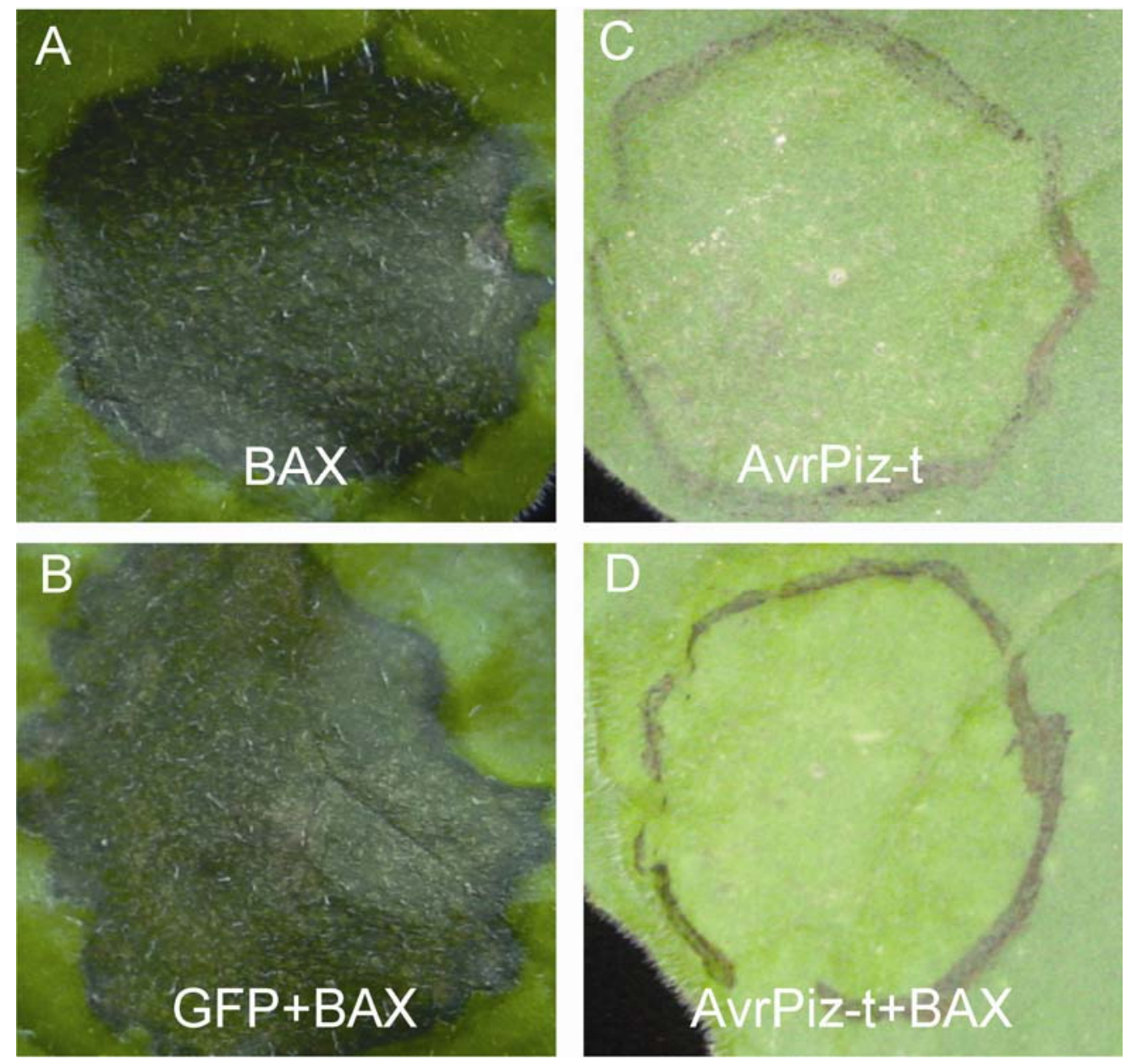

Fig. 4. Suppression of BAX-mediated programmed cell death (PCD) by AvrPiz-t in Nicotiana benthamiana leaves using Agrobacterium infiltration. The photographs of a representative leaf were shown. A, BAX triggered the PCD. B, Green fluorescent protein (GFP) did not interfere with the BAX-mediated PCD. C, AvrPiz-t alone did not induce the cell death. D, AvrPiz-t significantly suppressed the BAX-mediated PCD. 
Screen of the BAC clone by pool method.

The whole BAC library of 81278ZB15 saved in 24 384-well plates was divided into 24 pools (i.e., one pool per 384 -well plate) and DNA from each pool was isolated for the first round of screening. The markers proximal to or co-segregating with AvrPiz-t were used to screen the positive pools by PCR method. Each of the 384-well plates corresponding to a positive pool was further divided into four ordered 96-well plates. The DNA was isolated from each 96-well plate and was saved as subpools. The subpools were used for the second screen with the same markers by PCR method. At the final step, the 96-well plate corresponding to the positive subpool was divided into 20 DNAs based on the 12-by-8 format for the isolation of a single positive BAC clone.

\section{BAC clone sequencing, sequence assembly, and gene prediction.}

A BAC clone, BAC07bg07, identified by the pool method as described above was selected for sequencing using a shot-gun approach as described previously (Zhou et al. 2006). More than 1,200 subclone ends were sequenced and processed for sequence assembly using the software packages Phred/Phrap/ Gap4 (Ewing and Green 1998; Ewing et al. 1998). Gaps present in the assembled sequence were further filled as described previously (Zhou et al. 2006). Genes of the derived BAC sequence were predicted using the Fgenesh program.

\section{Vector modification for fungal transformation.}

A fragment containing the ccdB gene and chlorophenol resistance gene from the gateway destination vector pPANDA developed by Miki and Shimamoto (2004) was amplified by PCR with the following primers: forward, 5'-ggcgcgccatttttt aatggcggccgcattaggcacc- $3^{\prime}$ and reverse, 5' -ttaattaaccattttttaat ggtcgacctgcagactggctg- $3^{\prime}$, with the restriction sites for AscI and PacI underlined, and cloned into pGEMT-easy vector (Promega Corp., Madison, WI, U.S.A.). The derived plasmid was designated pGEM-T-ncgr. A fragment released from pGEM-T-attR by digestion with EcoRI was further cloned into pBluescript KSII predigested with the same enzyme and the derived vector was named PKS-ncgr. A fragment released from PKS-ncgr by digestion with HindIII and SacI was finally cloned into the vector pCSN43 developed by Staben and associates (1989). The derived vector for M. oryzae transformation was designated pCSN43-ncgr.

\section{Fungal transformation and complementation test.}

All the constructs listed in Table 1 were subcloned into pCSN43-ncgr because the modified vector is highly efficient for cloning, with two 8-nucleotide restriction enzymes, AscI and PacI, introduced into the multiple cloning sites. The DNA corresponding to each candidate gene was amplified by PCR using pfu polymerase (Promega Corp.) with the primers listed in Supplementary Table 3 . The amplified fragment either was cloned into pGEM-T-attR to introduce $A s c \mathrm{I}$ and PacI for subsequent cloning in pCSN43-ncgr, or directly cloned into pCSN43-ncgr by digestion with the appropriate restriction enzymes to obtain the constructs for the complementation tests (Table 2). All the constructs were confirmed by sequencing. Fungal transformation was performed by a polyethylene glycol (PEG)-mediated approach as described previously (Sweigard et al. 1995). The virulent isolate GUY11 was used as the receipt isolate for transformations in this study. Five independent hygromycin-resistant transformants were selected for pathogenicity tests.

\section{Sequence analysis of virulent and avirulent isolates.}

The sequences of AvrPiz-t, including 5' regulatory and coding sequences, were amplified by PCR with the following primers: forward, 5'-tttaaattaagcttccaacgtagagcgtatgc-3' and reverse, 5'aaatttgagctctccgttgaagcaacaggatc- $3^{\prime}$. The amplified DNA was analyzed by electrophoresis on a $1 \%$ agarose gel to determine any visible size difference compared with the one amplified from the wild-type strain. The AvrPiz-t coding sequence from each tested isolate was completely determined for sequence comparison.

\section{A. tumefaciens infiltration assays.}

We adopted the Agrobacterium-mediated transient expression system as described previously (Bos et al. 2006) to analyze whether AvrPiz-t was able to suppress the BAX-mediated PCD. The 35S:AvrPiz-t construct was generated by inserting a fragment encompassing the complete AvrPiz-t ORF amplified by PCR from isolate $70-15$ of $M$. oryzae using the primers AvrPiz-tF (5' -ccatcgatATGAGCTTCGTACAATGCAATC-3', ClaI site underlined) and AvrPiz-tR (5'-cggtcgacCTATTGG CGCTGAGCCTGAGGG-3', SalI site underlined) between the $C l a \mathrm{I}$ and SalI sites in the Potato virus $X$ binary vector pGR106 (pGR106:AvrPiz-t). Similarly, BAX was cloned into pGR106 (pGR106:BAX) after being amplified using primers BAXF (5'ccatcgatATGGACGGGTCCGGGGAG-3', ClaI site underlined) and BAXR (5'-cggtcgacTCAGCCCATCTTCTTCCAG-3', SalI site underlined). After verification by sequencing, the constructs were introduced into the A. tumefaciens GV2260. Overnight cultures in Luria-Bertani media supplemented with kanamycin at $50 \mathrm{mg} / \mathrm{ml}$ were harvested by centrifugation. The harvested cells were washed three times in $10 \mathrm{mM} \mathrm{MgCl}$ and resuspended in $10 \mathrm{mM} \mathrm{MgCl}$ to an optical density at $600 \mathrm{~nm}$ of 0.3. After incubation at room temperature for $2 \mathrm{~h}$, the cells were used to infiltrate into 4 - to 6 -week-old $N$. benthamiana leaves using a 1-ml needleless syringe as described previously (Romeis et al. 2001). Plants were grown and maintained throughout the experiments in a greenhouse with an ambient temperature of $25^{\circ} \mathrm{C}$ and $12 \mathrm{~h}$ of day light with high light intensity. A. tumefaciens cells carrying the BAX gene (pGR106:BAX) were infiltrated into the same site $24 \mathrm{~h}$ later. As controls, A. tumefaciens strains carrying BAX or AvrPiz-t were replaced with A. tumefaciens strains carrying the GFP gene (pGR106:GFP). Symptom development was monitored from 3 to 8 days after infiltration, and pictures were taken after 8 days. The experiments were repeated at least three times. Although it is likely that PVX replication occurred in the transformed plant cells, resulting in amplified expression of the genes in the PVX vector, no attempt was made to quantitate PVX replication in this experiment.

\section{ACKNOWLEDGMENTS}

This manuscript was selected as a feature article through the APS/CSPP Cooperative Agreement. We gratefully acknowledge T. Mitchell for his critical reading of the manuscript. We also thank X. Zhu at the Institute of Guangdong Academy of Agriculture, China for providing us with the set of the isolates as listed in Table 3; and H. Leung at the International Rice Research Institute for providing us with the Piz-t donor line IRBL11. This research was supported by grants from the National "863" High-Tech Project of China (2006AA10A102), the Commonweal Specialized Research Fund of China Agriculture (200803008), and "973" Programme (2006CB101901) to B. Zhou; and National Science foundation of China (30400260) and Key Project of Fujian Natural Science Foundation (B0520002) to Z. Wang.

\section{LITERATURE CITED}

Alfano, J. R., and Collmer, A. 2004. Type III secretion system effector proteins: Double agents in bacterial disease and plant defense. Annu. Rev. Phytopathol. 42:385-414.

Allen, R. L., Bittner-Eddy, P. D., Grenvitte-Briggs, L. J., Meitz, J. C., Rehmany, A. P., Rose, L. E., and Beynon, J. L. 2004. Host-parasite 
coevolutionary conflict between Arabidopsis and downy mildew. Science 306:1957-1960.

Armstrong, M. R., Whisson, S. C., Pritchard, L., Bos, J. I., Venter, E., Avrova, A. O., Rehmany, A. P., Böhme, U., Brooks, K., Cherevach, I., Hamlin, N., White, B., Fraser, A., Lord, A., Quail, M. A., Churcher, C., Hall, N., Berriman, M., Huang, S., Kamoun, S., Beynon, J. L., and Birch, P. R. 2005. An ancestral oomycete locus contains late blight avirulence gene Avr3a, encoding a protein that is recognized in the host cytoplasm. Proc. Natl. Acad. Sci. U.S.A. 102:7766-7771.

Böhnert, H. U., Fudal, I., Dioh, W., Tharreau, D., Notteghem, J. L., and Lebrun, M. H. 2004. A putative polyketide synthase/peptide synthetase from Magnaporthe grisea signals pathogen attack to resistant rice. Plant Cell 16:2499-2513.

Bonman, J. M., Vergel de Dios, T. I., Bandong, J. M., and Lee, E. J. 1987. Pathogenic variability of monoconidial isolates of Pyricularia oryzae in Korea and in the Philippines. Plant Dis. 71:127-130.

Bos, J. I., Kanneganti, T. D., Young, C., Cakir, C., Huitema, E., Win, J., Armstrong, M. R., Birch, P. R., and Kamoun, S. 2006. The C-terminal half of Phytophthora infestans RXLR effector AVR3a is sufficient to trigger R3a-mediated hypersensitivity and suppress INF1-induced cell death in Nicotiana benthamiana. Plant J. 48:165-176.

Bryan, G. T., Wu, K. S., Farrall, L., Jia, Y., Hershey, H. P., McAdams, S. A., Faulk, K. N, Donaldson, G. K, Tarchini, R., and Valent, B. 2000. A single amino acid difference distinguishes resistant and susceptible alleles of the rice blast resistance gene Pi-ta. Plant Cell 12:2033-2045.

Catanzariti, A. M., Dodds, P. N., Lawrence, G. J., Ayliffe, M. A., and Ellis, J. G. 2006. Haustorially expressed secreted proteins from flax rust are highly enriched for avirulence elicitors. Plant Cell 18:243-256.

Chadha, S., and Gopalakrishna, T. 2005. Retrotransposon-microsatellite amplified polymorphism (REMAP) markers for genetic diversity assessment of the rice blast pathogen (Magnaporthe grisea). Genome 48:943-945.

Chen, X., Shang, J., Chen, D., Lei, C., Zou, Y., Zhai, W., Liu, G., Xu, J., Ling, Z., Cao, G., Ma, B., Wang, Y., Zhao, X., Li, S., and Zhu, L. 2006. A B-lectin receptor kinase gene conferring rice blast resistance. Plant J. 46:794-804.

Chisholm, S. T., Coaker, G., Day, B., and Staskawicz, B. J. 2006. Hostmicrobe interactions: Shaping the evolution of the plant immune response. Cell 124:803-814.

Dai, L. Y., Liu, X. L., Xiao, Y. H., and Wang, G. L. 2007. Recent advances in cloning and characterization of disease resistance genes in rice. $\mathrm{J}$. Integr. Plant Biol. 49:112-119.

Dangl, J. L., and Jones, J. D. G. 2001. Plant pathogens and integrated defence responses to infection. Nature 411:826-833.

Dean, R. A., Talbot, N. J., Ebbole, D. J., Farman, M. L., Mitchell, T. K., Orbach, M. J., Thon, M, Kulkarni, R, Xu, J. R., Pan, H., Read, N. D., Lee, Y. H., Carbone, I., Brown, D., Oh, Y. Y., Donofrio, N., Jeong, J. S., Soanes, D. M., Djonovic, S., Kolomiets, E., Rehmeyer, C., Li, W., Harding, M., Kim, S., Lebrun, M. H., Böhnert, H., Coughlan, S., Butler, J., Calvo, S., Ma, L. J., Nicol, R., Purcell, S., Nusbaum, C., Galagan, J. E., and Birren, B. W. 2005. The genome sequence of the rice blast fungus Magnaporthe grisea. Nature 434:980-986.

de Torres, M., Mansfield, J. W., Grabov, N., Brown, I. R., Ammouneh, H., Tsiamis, G., Forsyth, A., Robatzek, S., Grant, M., and Boch, J. 2006. Pseudomonas syringae effector AvrPtoB suppresses basal defence in Arabidopsis. Plant J. 47:368-382.

Dodds, P. N., Lawrence, G. J., Catanzariti, A. M., Ayliffe, M. A., and Ellis, J. G. 2004. The Melampsora lini AvrL567 avirulence genes are expressed in haustoria and their products are recognized inside plant cells. Plant Cell 16:755-768.

Dou, D. L., Kale, S. D., Wang, X. L., Chen, Y. B., Wang, Q. Q., Wang, X., Jiang, R. H., Arredondo, F. D., Anderson, R. G., Thakur, P. B., McDowell, J. M., Wang, Y., and Tyler, B. M. 2008. Conserved C-terminal motifs required for avirulence and suppression of cell death by Phytophthora sojae effector Avr1b. Plant Cell 20:1118-1133.

Ewing, B., and Green, P. 1998. Base calling of automated sequencer traces using Phred. II. error probabilities. Genome Res. 8:186-194.

Ewing, B., Hillier, L., Wendl, M. C., and Green, P. 1998. Base calling of automated sequencer traces using Phred. I accuracy assessment. Genome Res. 8:175-185.

Farman, M., Taura, S., and Leong, S. A. 1996. The Magnaporthe grisea DNA fingerprinting probe MGR586 contain the $3^{\prime}$ end of an inverted repeat transposon. Mol. Gen. Genet. 251:675-681.

Farman, M. L., Eto, Y., Nakao, T., Tosa, Y., Nakayashiki, H., Mayama, S., and Leong, S. A. 2002. Analysis of the structure of the AVR1-CO39 avirulence locus in virulent rice-infecting isolates of Magnaporthe grisea. Mol. Plant-Microbe Interact. 15:6-16.

Flor, H. 1971. Current status of the gene-for-gene concept. Annu. Rev. Phytopathol. 9:275-296.

Grant, S. R., Fisher, E. J., Chang, J. H., Mole, B. M., and Dangl, J. L.
2006. Subterfuge and manipulation: Type III effectors of phytopathogenic bacteria. Annu. Rev. Microbiol. 60:425-449.

He, P., Shan, L., Lin, N. C., Martin, G. B., Kemmerling, B., Nurnberger, T., and Sheen, J. 2006. Specific bacterial suppressors of MAMP signaling upstream of MAPKKK in Arabidopsis innate immunity. Cell 125:563-575.

International Rice Genome Sequencing Project. 2005. The map-based sequence of the rice genome. Nature 436:793-800.

Jamir, Y., Guo, M., Oh, H. S., Petnicki-Ocwieja, T., Chen, S., Tang, X., Dickman, M. B., Collmer, A., and Alfano, J. R. 2004. Identification of Pseudomonas syringae type III effectors that can suppress programmed cell death in plants and yeast. Plant J. 37:554-565.

Jia, Y., McAdams, S. A., Bryan, G. T., Hershey, H. P., and Valent, B. 2000. Direct interaction of resistance gene and avirulence gene products confers rice blast resistance. EMBO (Eur. Mol. Biol. Organ.) J. 19:4004-4014.

Jones, J. D., and Dangl, J. L. 2006. The plant immune system. Nature 444:323-329.

Kamoun, S. 2006. A catalogue of the effector secretome of plant pathogenic oomycetes. Annu. Rev. Phytopathol. 44:41-60.

Kamoun, S. 2007. Groovy times: Filamentous pathogen effectors revealed. Curr. Opin. Plant Biol. 10:358-365.

Kang, S., Sweigard, J. A., and Valent, B. 1995. The $P W L$ host specificity gene family in the blast fungus Magnaporthe grisea. Mol. PlantMicrobe Interact. 8:939-948.

Kang, S., Lebrum, M. H., Farrall, L., and Valent, B. 2001. Gain of virulence caused by insertion of a Pot3 transposon in a Magnaporthe grisea avirulence gene. Mol. Plant-Microbe Interact. 14:671-674.

Khang, C. H., Park, S. Y., Lee, Y. H., Valent, B., and Kang, S. 2008. Genome organization and evolution of the AVR-Pita avirulence gene family in the Magnaporthe grisea species complex. Mol. Plant-Microbe Interact. 21:658-670.

Kim, M. G., da Cunha, L., McFall, A. J., Belkhadir, Y., DebRoy, S., Dangl, J. L., and Mackey, D. 2005. Two Pseudomonas syringae type III effectors inhibit RIN4-regulated basal defense in Arabidopsis. Cell 121:749-759.

Kiyosawa, S. 1976. Pathogenic variations of Pyricularia oryzae and their use in genetic and breeding studies. SABRAO J. 8:53-67.

Li, X., Lin, H., Zhang, W., Zou, Y., Zhang, J., Tang, X., and Zhou, J. M. 2005. Flagellin induces innate immunity in nonhost interactions that is suppressed by Pseudomonas syringae effectors. Proc. Natl. Acad. Sci. U.S.A. 102:12990-12995.

Lin, F., Chen, S., Que, Z., Wang, L., Liu, X., and Pan, Q. 2007. The blast resistance gene $\mathrm{Pi} 37$ encodes a nucleotide binding site-leucine-rich repeat protein and is a member of a resistance gene cluster on rice chromosome 1. Genetics 177:1871-1880.

Liu, X., Lin, F., Wang, L., and Pan, Q. 2007. The in silico map-based cloning of Pi36, a rice coiled-coil-nucleotide-binding site-leucine-rich repeat gene that confers race-specific resistance to the blast fungus. Genetics 176:2541-2549.

Luo, C. X., Yin, L. F., Koyanagi, S., Farman, M. L., Kusaba, M., and Yaegashi, H. 2005. Genetic mapping and chromosomal assignment of Magnaporthe oryzae avirulence genes AvrPik, AvrPiz, and AvrPiz-t controlling cultivar specificity on rice. Phytopathology 95:640-647.

Ma, J. H., Wang, L., Feng, S. J., Lin, F., Xiao, Y., and Pan, Q. H. 2006. Identification and fine mapping of AvrPi15, a novel avirulence gene of Magnaporthe grisea. Theor. Appl. Genet. 113:875-883.

Martin, S. L., Blackmon, B. P., Rajagopalan, R., Houfek, T. D., Sceeles, R. G., Denn, S. O., Mitchell, T. K., Brown, D. E., Wing, R. A., and Dean, R. A. 2002. MagnaportheDB: A federated solution for integrating physical and genetic map data with BAC end derived sequences for the rice blast fungus Magnaporthe grisea. Nucleic Acid Res. 30:121-124.

McHale, L., Tan, X., Koehl, P., and Michelmore, R. W. 2006. Plant NBSLRR proteins: Adaptable guards. Genome Biol. 7:212.

Meyers, B. C., Kozik, A., Griego, A., Kuang, H., and Michelmore, R. W. 2003. Genome-wide analysis of NBS-LRR-encoding genes in Arabidopsis. Plant Cell 15:809-834.

Miki, D., and Shimamoto, K. 2004. Simple RNAi vectors for stable and transient suppression of gene function in rice. Plant Cell Physiol. 45:490-495.

Nishimura, M., Hayashi, N., Jwa, N. S., Lau, G. W., Hamer, J. E., and Hasebe, A. 2000. Insertion of the LINE retrotransposon MGL causes a conidiophore pattern mutation in Magnaporthe grisea. Mol. PlantMicrobe Interact. 13:892-894.

Nomura, K., DebRoy, S., Lee, Y. H., Pumplin, N., Jones, J., and He, S. Y. 2006. A bacterial virulence protein suppresses host innate immunity to cause plant disease. Science 313:220-223.

Oh, H. S., and Collmer, A. 2005. Basal resistance against bacteria in Nicotiana benthamiana leaves is accompanied by reduced vascular staining and suppressed by multiple Pseudomonas syringae type III secretion system effector proteins. Plant J. 44:48-59.

Orbach, M. J., Farrall, L., Sweigard, J. A., Chrmley, F. G., and Valent, B. 
2000. A telomeric avirulence gene determines efficacy for the rice blast resistance gene Pi-ta. Plant Cell 12:2019-2032.

Peyyala, R., and Farman M. L. 2006. Magnaporthe oryzae isolates causing gray leaf spot of perennial ryegrass possess a functional copy of the AVR1-CO39 avirulence gene. Mol. Plant Pathol. 7:157-165.

Qu, S., Liu, G., Zhou, B., Bellizzi, M., Zeng, L., Dai, L., Han, B., and Wang, G. L. 2006. The broad-spectrum blast resistance gene Pi9 encodes an NBS-LRR protein and is a member of the multiple family in rice. Genetics 172:1901-1914.

Rehmany, A. P., Gordon, A., Rose, L. E., Allen, R. L., Armstrong, M. R., Whisson, S. C., Kamoun, S., Tyler, B. M., Birch, P. R., and Beynon, J. L. 2005. Differential recognition of highly divergent downy mildew avirulence gene alleles by RPP1 resistance genes from two Arabidopsis lines. Plant Cell 17:1839-1850.

Romeis, T., Ludwig, A. A., Martin, R., and Jones, J. D. 2001. Calciumdependent protein kinases play an essential role in a plant defence response. EMBO (Eur. Mol. Biol. Organ.) J. 20:5556-5567.

Shan, W., Cao, M., Leung, D., and Tyler, B. M. 2004. The Avrlb locus of Phytophthora sojae encodes an elicitor and a regulator required for avirulence on soybean plants carrying resistance gene Rps $1 b$. Mol. Plant-Microbe Interact. 17:394-403.

Staben, C., Jensen, B., Singer, M., Pollock, J., Schechtman, M., Kinsey, J., and Selker, E. 1989. Use of a bacterial Hygromycin B resistance gene as a dominant selectable marker in Neurospora crassa transformation. Fungal Genet. Newsl. 36:79-81.

Sweigard, J. A., Carroll, A. M., Kang, S., Farrall, L., Chumley, F. G., and Valent, B. 1995. Identification, cloning, and characterization of PWL2, a gene for host species specificity in the rice blast fungus. Plant Cell 7:1221-1233

Thon, M. R., Pan, H., Diener, S., Papalas, J., Taro, A., Mitchell, T. K., and Dean, R. A. 2006. The role of transposable element clusters in genome evolution and loss of synteny in the rice blast fungus Magnaporthe oryzae. Genome Biol. 7:R16.

Underwood, W., Zhang, S., and He, S. Y. 2007. The Pseudomonas syrin- gae type III effector tyrosine phosphatase HopAO1 suppresses innate immunity in Arabidopsis thaliana Plant J. 52:658-672.

Valent, B. 1990. Rice blast as a model system for plant pathology. Phytopathology 80:33-36.

Wang, B. H., Lu, G. D., Lin, W. M., and Wang, Z. H. 2002. Genetic analysis and molecular marker of Avr-Pi1, Avr-Pi2 and Avr-Pi4a of Magnaporthe grisea. Yi Chuan Xue Bao 29:820-826.

Wang, Z. X., Yano, M., Yamanouchi, U., Iwamoto, M., Monna, L. Hayasaka, H., Katayose, Y., and Sasaki, T. 1999. The Pib gene for rice blast resistance belongs to the nucleotide binding and leucine-rich repeat class of plant disease resistance genes. Plant J. 19:55-64.

Zhou, B., Qu, S., Liu, G., Dolan, M., Sakai, H., Lu, G., Bellizzi, M., and Wang, G. L. 2006. The eight amino-acid differences within three leucine-rich repeats between Pi2 and Piz-t resistance proteins determine the resistance specificity to Magnaporthe grisea. Mol. Plant-Microbe Interact. 19:1216-1228.

Zhou, E., Jia, Y., Singh, P., Correll, J. C., and Lee, F. N. 2007. Instability of the Magnaporthe oryzae avirulence gene AVR-Pita alters virulence. Fungal Genet. Biol. 44:1024-1034.

Zipfel, C., Robatzek, S., Navarro, L., Oakeley, E. J., Jones, J. D., Felix, G. and Boller, T. 2004. Bacterial disease resistance in Arabidopsis through flagellin perception. Nature 428:764-767

Zipfel, C., Kunze, G., Chinchilla, D., Caniard, A., Jones, J. D., Boller, T. and Felix, G. 2006. Perception of the bacterial PAMP EF-Tu by the receptor EFR restricts Agrobacterium-mediated transformation. Cell 125:749-760.

\section{AUTHOR-RECOMMENDED INTERNET RESOURCES}

SignalP 3.0server: www.cbs.dtu.dk/services/SignalP

Softberry ProtComp and Fgenesh programs: www.softberry.com Magnaporthe grisea database:

www.broad.mit.edu/annotation/fungi/magnaporthe 